\title{
Expresión de receptores hormonales como factor predictivo de respuesta al tratamiento en pacientes con cáncer de mama temprano y localmente avanzado con sobreexpresión de HER2 en un hospital privado
}

\section{The expression of hormone receptors as a predictive factor of response to treatment in patients with early and locally advanced breast cancer with HER2 overexpression in a private hospital}

\author{
Raúl Alejandro Andrade-Moreno, ${ }^{*}$ Geovani Amador-García, ${ }^{*}$ \\ José Fabián Martínez-Herrera, ${ }^{*, \neq}$ Guillermo Manuel Olivares-Beltrán ${ }^{*}$
}

Citar como: Andrade-Moreno RA, Amador-García G, Martínez-Herrera JF, Olivares-Beltrán GM. Expresión de receptores hormonales como factor predictivo de respuesta al tratamiento en pacientes con cáncer de mama temprano y localmente avanzado con sobreexpresión de HER2 en un hospital privado. An Med ABC. 2021; 66 (4): 258-262. https://dx.doi.org/10.35366/102925

\section{RESUMEN}

Introducción: En la actualidad, el cáncer de mama es la primera causa de muerte asociada al cáncer en mujeres. Uno de múltiples subtipos de esta enfermedad, conocido por la sobreexpresión del receptor del factor de crecimiento epidermoide humano 2 (HER2), tiene un mal pronóstico. El desarrollo y uso de trastuzumab ha mejorado significativamente los desenlaces oncológicos; sin embargo, no todos los casos se benefician de este tratamiento. Objetivo: Comparar la tasa de respuesta patológica completa posterior al tratamiento neoadyuvante en pacientes mexicanas con cáncer de mama HER2+ localmente avanzado, y su relación con la expresión de receptores hormonales. Material y métodos: Estudio descriptivo de una cohorte de carácter retrospectivo, unicéntrico de casos con cáncer de mama localmente avanzado y temprano receptor del factor de crecimiento epidermoide humano $2+$ tratadas con quimioterapia y tratamiento antirreceptor

\section{ABSTRACT}

Introduction: Breast cancer is the leading cause of cancerassociated death in women. One of the multiple subtypes of this disease, known for the overexpression of the human epidermal growth factor receptor 2 (HER2) has a poor prognosis. The development of trastuzumab has significantly improved cancer outcomes in these patients, however, not all patients benefit from this therapy. Objective: To compare the rate of pathological complete response after neoadjuvant treatment in Mexican patients with early and locally advanced HER2+ breast cancer, and its relationship with the expression of hormone receptors. Material and methods: A descriptive study of a retrospective, single-center cohort of patients with early and locally advanced HER2 + breast cancer treated with chemotherapy and antiHER2 therapy in the ABC Medical Center. Results: 35 patients were included in the study. The pathological complete response
* Departamento de Oncología Médica. Centro Médico ABC, Ciudad de México.

‡ Latin American Network for Cancer Research (LAN-CANCER), Lima, Perú.

Recibido: 11/05/2021. Aceptado: 26/11/2021.
Correspondencia:

Raúl Alejandro Andrade-Moreno

E-mail: randradem.md@gmail.com 
del factor de crecimiento epidermoide humano 2 en el Centro Médico ABC. Resultados: Se incluyeron 35 casos en el estudio. La respuesta patológica completa fue alcanzada en $60 \%$ de la población general (21 pacientes), con un porcentaje más alto de aquéllos que alcanzaron una respuesta patológica completa en el grupo con receptores hormonales negativos a comparación de aquéllos con expresión de receptores hormonales (83 vs 48\%). Conclusiones: En el cáncer de mama con sobreexpresión del receptor del factor de crecimiento epidermoide humano 2 localmente avanzado y temprano, la falta de expresión de receptores hormonales está asociada a una mayor sensibilidad al tratamiento neoadyuvante, determinado por un mayor número de pacientes que alcanzan una respuesta patológica completa.

Palabras clave: Cáncer de mama, HER2, neoadyuvancia, respuesta patológica completa.

Nivel de evidencia: IV was achieved in $60 \%$ of the general population (21 patients). A higher percentage of patients achieving a complete pathological response was identified in the group of hormone receptor negative patients compared to hormone receptor positive patients. (83 versus 48\%). Conclusion: In early and locally advanced stage HER2 + breast cancer, the lack of expression of hormone receptors is associated with a greater sensitivity to neoadjuvant treatment, determined by a greater percentage of patients who achieve a pathological complete response.

Keywords: Breast cancer, HER2, Neoadjuvant treatment, pathological complete response.

Level of evidence: IV

\author{
Abreviaturas: \\ $\mathrm{AC} / \mathrm{THP}=$ Adriamicina, ciclofosfamida, paclitaxel, trastuzumab, pertuzumab. \\ HER2 = Receptor del factor de crecimiento epidermoide humano 2. \\ $\mathrm{pCR}=$ Respuesta patológica completa. \\ $\mathrm{RH}=$ Receptores hormonales. \\ $S G=$ Supervivencia global \\ SLE = Supervivencia libre de enfermedad. \\ THP = Docetaxel, trastuzumab, pertuzumab. \\ $\mathrm{TCHP}=$ Docetaxel, carboplatino, trastuzumab, pertuzumab.
}

\section{INTRODUCCIÓN}

El cáncer de mama es la neoplasia maligna más frecuente en la mujer y la primera causa de muerte asociada. ${ }^{1}$ Esta enfermedad es heterogénea, fenotípicamente diversa y compuesta de distintos subtipos biológicos. ${ }^{2}$ Uno de estos subtipos se caracteriza por la amplificación o sobreexpresión de receptor del factor de crecimiento epidermoide humano 2 (HER2), un receptor crítico en la activación de vías de crecimiento y diferenciación celular, por lo que se considera como una enfermedad agresiva, con altas tasas de recurrencia y diseminación sistémica. ${ }^{3,4}$ Este subtipo se expresa en alrededor de $23 \%$ de la población mexicana. ${ }^{5}$

Gracias al desarrollo del trastuzumab, un anticuerpo monoclonal específico contra el receptor de crecimiento epidermoide 2 , el pronóstico de las pacientes con cáncer de mama HER2 + ha mejorado notoriamente, y en la actualidad se cuentan con múltiples opciones de tratamiento. ${ }^{6}$ Gracias a este descubrimiento, en poco menos de una década el número y la calidad de los tratamientos dirigidos contra HER2, entre ellos el pertuzumab, el pronóstico de estas pacientes ha mejorado significativamente. ${ }^{7}$

El estándar de tratamiento para estas pacientes en etapa temprana y localmente avanzada incluye el uso combinado de quimioterapia con trastuzumab y pertuzumab, administrado de forma previa al tratamiento quirúrgico (neoadyuvante) y posterior a él (adyuvante). 8,9

El beneficio de la doble terapia como componente del tratamiento neoadyuvante en el subtipo HER2 está bien fundamentado en ensayos clínicos y metaanálisis que muestran un incremento en la respuesta patológica completa (pCR), es decir, a la ausencia de enfermedad invasiva residual en la mama y en los ganglios axilares muestreados de la enfermedad. El alcanzar esta respuesta posterior al tratamiento, a pesar de no considerarse un subrogado de supervivencia global, ha correlacionado con una mejoría en las tasas de supervivencia libre de enfermedad; ${ }^{10-12}$ sin embargo, los resultados de su eficacia y los factores predictivos en población mexicana son escasos y se conocen pocas estadísticas.

Objetivo: comparar la respuesta patológica completa posterior al tratamiento neoadyuvante con terapia anti-HER2 y quimioterapia en una población mexicana no seleccionada en etapa temprana y localmente avanzada con cáncer de mama HER2+ y receptores hormonales $(\mathrm{RH})$ contra aquéllos que no expresan estos últimos.

\section{MATERIAL Y MÉTODOS}

Análisis retrospectivo de expedientes médicos de casos con cáncer de mama HER2+ en etapa temprana o localmente avanzada y que fueron consideradas candidatas para tratamiento neoadyuvante. Todas recibieron tratamiento sistémico con pertuzumab y trastuzumab en asociación con quimioterapia de 
Tabla 1: Variables demográficas y clínico-patológicas población neoadyuvante. $\mathrm{N}=35$.

\begin{tabular}{|c|c|}
\hline Característica & $\mathrm{n}(\%)$ \\
\hline \multicolumn{2}{|l|}{ Edad } \\
\hline Mediana & 47 años \\
\hline Rango & $28-79$ \\
\hline \multicolumn{2}{|l|}{ Índice de masa corporal } \\
\hline Normal & $21(60)$ \\
\hline Sobrepeso u obesidad & $14(40)$ \\
\hline \multicolumn{2}{|l|}{ Patología } \\
\hline Ductal & $32(91.4)$ \\
\hline Lobulillar & $3(8.6)$ \\
\hline \multicolumn{2}{|l|}{ Tamaño tumoral ${ }^{*}$} \\
\hline T1 & $7(20.0)$ \\
\hline $\mathrm{T} 2$ & $18(51.4)$ \\
\hline T3 & $8(22.9)$ \\
\hline $\mathrm{T} 4$ & $2(5.7)$ \\
\hline \multicolumn{2}{|l|}{ Ganglios* } \\
\hline No & $10(28.6)$ \\
\hline $\mathrm{N} 1$ & $17(48.6)$ \\
\hline N2 & $5(14.3)$ \\
\hline N3 & $3(8.6)$ \\
\hline \multicolumn{2}{|l|}{ Estadio clínico* } \\
\hline$\| A$ & $12(34.3)$ \\
\hline IIB & $11(31.4)$ \\
\hline IIIA & $8(22.9)$ \\
\hline IIIB & $1(2.9)$ \\
\hline IIIC & $3(8.6)$ \\
\hline \multicolumn{2}{|l|}{ Receptores hormonales } \\
\hline Positivo & $22(62.9)$ \\
\hline Negativo & $13(37.1)$ \\
\hline \multicolumn{2}{|l|}{ HER2 } \\
\hline Inmunohistoquímica & $31(88.6)$ \\
\hline $\begin{array}{l}\text { Hibridación fluorescente in } \\
\text { situ }\end{array}$ & $4(11.4)$ \\
\hline \multicolumn{2}{|l|}{ Ki67 } \\
\hline$>20$ & $27(77.1)$ \\
\hline$<20$ & $8(22.9)$ \\
\hline \multicolumn{2}{|l|}{ Quimioterapia } \\
\hline Antraciclina + taxano & $21(60.0)$ \\
\hline Taxano & $14(40.0)$ \\
\hline Platino & $6(17.1)$ \\
\hline
\end{tabular}

acuerdo con las guías internacionales. La evaluación patológica de las neoplasias fue realizada previa al inicio del tratamiento sistémico y posterior al tratamiento oncológico y a la cirugía. La respuesta patológica completa fue definida como ypT0/ypN0 (ausencia de enfermedad invasiva residual en la mama y en los ganglios axilares). Otras características histopatológicas incluyeron la expresión de receptores de estrógeno, progesterona y Ki67. Los datos clínicos analizados incluyeron tamaño del tumor, edad, índice de masa corporal, y número de ganglios afectados.

Se consultó el registro de quimioterapia del Centro Médico ABC FARMIS para la identificación de los casos, así como los registros del departamento de patología y los expedientes electrónicos y físicos del Centro Médico ABC. Los factores clínico-patológicos fueron analizados con el uso de pruebas no paramétricas. Se utilizó la prueba de $\chi^{2}$ o la prueba de Fisher para analizar la correlación de la respuesta y las características clínicopatológicas Los datos fueron introducidos y analizados mediante el programa estadístico SPSS versión 21.

\section{RESULTADOS}

Se incluyeron 35 casos con cáncer de mama HER2+ temprano o localmente avanzado, diagnosticadas y tratadas en el Centro de Cáncer del Centro Médico ABC entre enero de 2014 y junio de 2020 (Tabla 1).

La mediana de edad de la población fue de 47 años (rango 28-79) e incluyó a 20 pacientes menores de 50 años ( $57 \%$ de la población total), $40 \%$ se clasificó como con sobrepeso u obesidad al momento del diagnóstico.

La histología predominante fue el carcinoma ductal infiltrante expresado en $91 \%$ de los pacientes. Los estadios clínicos más frecuentes fueron IIA, (34.3\%) IIB $(31.4 \%)$ y IIIA (22.9\%). La población incluyó pacientes sin afectación ganglionar clasificada como N0 (28.6\%). El $71.4 \%$ restante tenía algun grado de afectación ganglionar y fue clasificada como N1 (48.6\%), N2 $(14.3 \%)$ y N3 $(8.6 \%)$.

Tabla 2: Respuesta al tratamiento por expresión de receptores hormonales.

\begin{tabular}{cccc}
\hline & Sí & No & \\
$\mathrm{pCR}$ & $\mathrm{n}(\%)$ & $\mathrm{n}(\%)$ & $p$ \\
\hline $\mathrm{RH}(+)$ & $11(47.8)$ & $12(52.2)$ & $\mathbf{0 . 0 4 5}$ \\
$\mathrm{RH}(-)$ & $10(83.3)$ & $2(16.7)$ & \\
\hline
\end{tabular}

$\mathrm{RH}+=$ Receptores hormonales positivos, $\mathrm{RH}-=$ Receptores hormonales negativos. 
La mayoría de las pacientes tenían tumores que medían más de 2 centímetros al momento del diagnóstico T2 (51.4\%), T3 (22.9\%) y T4 (5.7\%) y sólo $20 \%$ contaban con un tumor menor de 2 centímetros y fueron clasificados como T1.

La mayoría de los pacientes $(77.1 \%)$ tenían un índice de proliferación alto expresado con un Ki67 > 20 .

Un total de 13 pacientes (37.1\%) no contaban con expresión de receptores hormonales, el resto (62.9\%) fueron categorizados como Triple Positivo.

$\mathrm{El}$ régimen más común de tratamiento fue $\mathrm{AC} /$ THP $(57.5 \%)$, y en total hasta $60 \%$ de los pacientes recibieron antraciclinas, en combinación con otros fármacos quimioterápicos, el resto de las pacientes recibieron esquemas basados en taxanos (THP) y taxanos y carboplatino (TCHP).

La pCR definida como definida como ypT0 ypN0 se logró en el 60\%. Los pacientes con ausencia en la expresión de $\mathrm{RH}$ lograron una pCR en $83.3 \%$ de los casos, frente a $47.8 \%$ de la población triple positiva. Esta diferencia en la respuesta fue estadísticamente significativa $(\mathrm{p}=0.045)$ (Tabla 2$)$.

La razón de probabilidades (OR) mostró la presencia de $\mathrm{RH}$ como un factor de riesgo para no alcanzar una pCR con una OR= 6.6 (IC 95\% 1.17-37.02).Debido al uso de diferentes esquemas de tratamiento neoadyuvante, se dividió a los pacientes en aquéllas que recibieron tratamiento con antraciclinas contra las que recibieron esquemas libres de éstas. El resultado mostró diferencias a favor del uso de antraciclinas; sin embargo, no alcanzó diferencia estadística ( $p=0.163$ ), con 14 pacientes que recibieron tratamiento con antraciclinas que alcanzaron una pCR $(66.7 \%)$ frente a sólo seis pacientes que consiguieron una pCR (42.9\%) y que no recibieron quimioterapia basada en éstas.

Los tumores no luminales HER2 + $(p=0.040)$ fueron predictores independientes de $\mathrm{pCR}$ en la regresión logística multivariante. Ninguna otra variante, como Ki67, índice de masa corporal, edad, tamaño del tumor, tipo de quimioterapia administrada y estado de los ganglios linfáticos, fue estadísticamente significativa (Tablas 3 y 4 ).

\section{DISCUSIÓN}

Los resultados de nuestro análisis mostraron que la presencia de receptores hormonales en el cáncer de mama subtipo HER2 impacta significativamente en la tasa de $\mathrm{pCR}(\mathrm{p}=0.045)$. Estos resultados provienen de una cohorte de pacientes no representativa de la Ciudad de México tratadas en una institución privada; sin embargo, cuentan con características simila- res en cuanto a la edad, la distribución de sobrepeso/ obesidad y estadio clínico con relación a otros estudios descriptivos de la población mexicana, no obstante, es importante mencionar que la mayoría de los estudios de referencia en la neoadyuvancia no incluyen dentro de su población mujeres latinoamericanas.

Para el análisis de la respuesta a la terapia neoadyuvante se eligió la definición más estricta de $\mathrm{pCR}$, definida como ypT0 ypN0, el cual fue alcanzado en $60 \%$ de toda la población incluida. Esta definición puede discriminar mejor entre pacientes con resultados favorables y desfavorables en los subtipos más agresivos (triple negativo y HER2), aunque existe discrepancia en las definiciones de pCR entre los estudios.

Como objetivo primario se observó que la erradicación de la neoplasia fue mayor en el grupo de casos con receptores hormonales negativos ( 83 vs $48 \%$ ). Esta diferencia es estadísticamente significativa y es superior a lo reportado en el ensayo NeoSphere $^{13}$ (63.2 vs $26 \%$ ), la corte de antraciclinas de TRYPHAENA ${ }^{14}$ (65 vs $48 \%$ ) y la cohorte libre de ellas del mismo estudio ( 83 vs $50 \%$ ), y es similar a lo reportado en el estudio TRAIN-215 (89 vs 51\%) de la cohorte de antraciclinas y su contraparte basada en esquemas de taxano y carboplatino ( 84 vs $55 \%$ ), mostrando a pesar de la heterogeneidad de esquemas de tratamiento una respuesta favorable en pacientes con receptores hormonales negativos, pero casi la mitad de las pacientes con expresión de receptores hormonales alcanzó una pCR, $(48 \%)$ lo cual indica un beneficio en la mayoría de la población estudiada.

Ninguna otra determinante clínica como índice de masa corporal, Ki-67, estado ganglionar o tamaño tumoral, mostró diferencia estadística en la respuesta al tratamiento neoadyuvante. Nuestro estudio tiene debilidades que deben ser consideradas al momento de interpretar los resultados: se trata del análisis retrospectivo de una población pequeña de un hospital privado, lo que corresponde a un sesgo de selección importante, por lo que los resultados solos son inferenciales. Por lo que una población mayor y una recolección de datos de otros centros oncológicos

Tabla 3: Análisis de regresión logística binario.

\begin{tabular}{lclc}
\hline \multicolumn{1}{c}{ Variable } & Odds Ratio & \multicolumn{1}{c}{ IC 95\% } & $p$ \\
\hline $\begin{array}{l}\text { Receptores hormonales } \\
\text { negativos }\end{array}$ & 7.49 & $(1.05-53.05)$ & 0.040 \\
Índice de masa corporal & 1.01 & $(0.18-5.71)$ & 0.988 \\
Ki-67 & 4.5 & $(0.65-30.87)$ & 0.125 \\
Ganglios positivos & 1.3 & $(0.20-9.22)$ & 0.751 \\
\hline
\end{tabular}


Tabla 4: Otras variables.

\begin{tabular}{|c|c|c|c|}
\hline & \multicolumn{2}{|c|}{ Respuesta patológica completa } & \multirow[b]{2}{*}{$p$} \\
\hline & $\begin{array}{c}\text { Sí } \\
\mathrm{n}(\%)\end{array}$ & $\begin{array}{c}\text { No } \\
n(\%)\end{array}$ & \\
\hline \multicolumn{4}{|l|}{ Ki67 } \\
\hline$>20$ & $7(38.9)$ & $14(82.4)$ & 0.221 \\
\hline$<20$ & $11(61.1)$ & $3(17.6)$ & \\
\hline \multicolumn{4}{|c|}{ Índice de masa corporal } \\
\hline$<25$ & $7(38.9)$ & $14(82.4)$ & 0.737 \\
\hline$>25$ & $11(61.1)$ & $3(17.6)$ & \\
\hline \multicolumn{4}{|c|}{ Tamaño tumoral* } \\
\hline $\mathrm{T} 1$ & $5(23.8)$ & $2(14.3)$ & 0.379 \\
\hline $\mathrm{T} 2$ & $11(52.4)$ & $7(50)$ & \\
\hline T3 & $4(19.0)$ & $4(28.6)$ & \\
\hline T4 & $1(4.8)$ & $1(7.1)$ & \\
\hline \multicolumn{4}{|c|}{ Ganglios* } \\
\hline NO & $6(28.6)$ & $4(28.6)$ & 0.913 \\
\hline N1 & $10(47.6)$ & $7(50)$ & \\
\hline N2 & $3(14.3)$ & $2(14.3)$ & \\
\hline N3 & $2(9.5)$ & $1(7.1)$ & \\
\hline
\end{tabular}

podrían brindar mayor validez a los resultados anteriormente expuestos.

\section{CONCLUSIONES}

En esta población mexicana con cáncer de mama HER2+, existe una diferencia significativa entre el porcentaje que logra una $\mathrm{pCR}$, favoreciendo a aquéllas con tumores que no expresan receptores hormonales en comparación con aquéllas que sí lo hacen. A pesar de esta diferencia, la mayoría de la población logra alcanzar este beneficio independientemente de su estado, con tasas de respuesta similares a los ensayos clínicos previamente descritos. No se asociaron otras variantes clínicas o patológicas que impactaran en la pCR.

\section{REFERENCIAS}

1. Siegel RL, Miller KD, Jemal A. Cancer statistics, 2018. CA Cancer J Clin. 2018; 68: 7-30.

2. Henry NL et al. Cancer de mama. En: Niederhuber JE, Armitage JO, Doroshow JH, Kastan, Tepper, JE (Eds). Abeloff oncología clínica. 6th ed. Elsevier, Barcelona, España 2020, 1560-1603.
3. Yarden Y, Sliwkowski MX. Untangling the ErbB signalling network. Nat Rev Mol Cell Biol. 2001; 2 (2): 127-137. doi: $10.1038 / 35052073$.

4. Slamon DJ, Clark GM, Wong SG, Levin WJ, Ullrich A, McGuire WL. Human breast cancer: correlation of relapse and survival with amplification of the HER-2/neu oncogene. Science. 1987; 235 (4785): 177-182.

5. Reynoso-Noverón N, Villareal-Garza C et al. Clinical and epidemiological profile of breast cancer in Mexico: results of the Seguro Popular. J Glob Oncol. 2017; 3(6): 757-764.

6. Slamon DJ, Leyland-Jones B, Shak S, Fuchs H, Paton V, Bajamonde A et al. Use of chemotherapy plus a monoclonal antibody against HER2 for metastatic breast cancer that overexpresses HER2. N Engl J Med. 2001; 344 (11): 783-792.

7. Baselga J, Albanell J, Molina MA, Arribas J. Mechanism of action of trastuzumab and scientific update. Semin Oncol. 2001; 28 (5 Suppl 16): 4-11.

8. Gianni L, Pienkowski T, Im YH, Roman L, Tseng LM, Liu MC et al. Efficacy and safety of neoadjuvant pertuzumab and trastuzumab in women with locally advanced, inflammatory, or early HER2- positive breast cancer (NeoSphere): a randomised multicentre, open-label, phase 2 trial. Lancet Oncol. 2012; 13 (1): 25-32.

9. Von Minckwitz G, Procter M, de Azambuja E, Zardavas D, Benyunes M, Viale G et al. Adjuvant pertuzumab and trastuzumab in early HER2-positive breast cancer. N Engl J Med. 2017; 377 (2): 122-131.

10. Von Minckwitz G, Untch M, Blohmer JU, Costa SD, Eidtmann H, Fasching PA et al. Definition and impact of pathologic complete response on prognosis after neoadjuvant chemotherapy in various intrinsic breast cancer subtypes. J Clin Oncol. 2012; 30 (15): 1796-1804.

11. Cortazar P, Zhang L, Untch M, Mehta K, Costantino JP, Wolmark $\mathrm{N}$ et al. Pathological complete response and longterm clinical benefit in breast cancer: the CTNeoBC pooled analysis. Lancet. 2014; 384 (9938): 164-172.

12. I-SPY2 Trial Consortium, Yee D, DeMichele AM, Yau C, Isaacs C, Symmans WF et al. Association of event-free and distant recurrence-free survival with individual-level pathologic complete response in neoadjuvant treatment of stages 2 and 3 breast cancer: three-year follow-up analysis for the I-SPY2 adaptively randomized clinical trial. JAMA Oncol. 2020; 6 (9): 1355-1362.

13. Gianni L, Pienkowski T, Im YH. 5-year analysis of neoadjuvant pertuzumab and trastuzumab in patients with locally advanced, inflammatory, or early-stage HER2positive breast cancer (NeoSphere): a multicentre, openlabel, phase 2 randomised trial. Lancet Oncol. 2016; 17 (6): 791-800.

14. Schneeweiss A, Chia S, Hickish T, Harvey V, Eniu A, Hegg $\mathrm{R}$ et al. Pertuzumab plus trastuzumab in combination with standard neoadjuvant anthracycline-containing and anthracycline-free chemotherapy regimens in patients with HER2-positive early breast cancer: a randomized phase II cardiac safety study (TRYPHAENA). Ann Oncol. 2013; 24 (9): 2278-2284.

15. Van Ramshorst MS, Van der Voort A, Van Werkhoven ED, Mandjes IA, Kemper I, Dezentjé VO et al. Neoadjuvant chemotherapy with or without anthracyclines in the presence of dual HER2 blockade for HER2-positive breast cancer (TRAIN-2): a multicentre, open-label, randomised, phase 3 trial. Lancet Oncol. 2018; 19 (12): 1630-1640.

16. Giuliano AE, Edge SB, Hortobagyi GN. Eighth edition of the AJCC cancer staging manual: breast cancer. Ann Surg Oncol. 2018; 25 (7): 1783-1778. 